\title{
Preventative Strategies in the Management of ROP: A Review of Literature
}

\author{
Irina Livshitz \\ University Hospitals Case Medical Center, Cleveland, USA \\ Email: irina.livshitz@uhhospitals.org
}

Received 14 April 2015; accepted 15 May 2015; published 20 May 2015

Copyright (C) 2015 by author and Scientific Research Publishing Inc.

This work is licensed under the Creative Commons Attribution International License (CC BY). http://creativecommons.org/licenses/by/4.0/

c) (†) Open Access

\begin{abstract}
Retinopathy of prematurity (ROP) is a potentially blinding eye disorder that primarily affects premature infants weighing 1250 grams or less that are born before 31 weeks of gestation. It is one of the most common causes of visual loss in childhood and can lead to lifelong vision impairment and blindness. Certain strategies for prevention have been confirmed by numerous trials, namely the role of oxygen therapy as an independent risk factor for the development of ROP and the benefits of strict control of premature infant oxygen saturations in the NICU. There is valuable data that supports use of other approaches, such as beta adrenergic blockade and IGF-1 supplementation, for which clinical trials are still in the works to establish clear protocols for their clinical use. Supplementation with vitamin A, omega-3 fatty acids, and inositol are all exciting arenas for further trials as preliminary data shows promising results in ROP prevention. It has also been shown that the benefit of vitamin $E$ is not worth the increased incidence of NEC and sepsis as potential side effects. Furthermore, while it was an interesting idea to use the chelator D-penicillamine as a preventative strategy for ROP, the results of multiple trials seemed to be equivocal. This brief review is not all-inclusive, as there are many more modes of prevention currently being studied.
\end{abstract}

\section{Keywords}

Retinopathy of Prematurity (ROP), Proliferative Retinopathy, Neonatology

\section{Introduction}

Retinopathy of prematurity (ROP) is a potentially blinding eye disorder that primarily affects premature infants weighing 1250 grams or less that are born before 31 weeks of gestation. It is one of the most common causes of visual loss in childhood and can lead to lifelong vision impairment and blindness [1]. We have progressed leaps 
and bounds in the realm of treatment for the proliferative phase of ROP. The standard of care at most institutions today is laser or cryo ablative therapy, which is unfortunately not always effective at preventing the sequelae of ROP and can lead to the loss of peripheral vision. This has sparked a new focus on anti-VEGF treatment, which itself carries a host of reservations because no one can yet be sure of the systemic effects of anti-VEGF in the neonate. For years, researchers have postulated that in order to tackle ROP and its potentially devastating consequences, we must consider preventative strategies rather than treatment alone. Such an approach could be of enormous benefit in providing a cheap, effective means to halt the disease in its tracks. What follows is a non-comprehensive review of modes of prevention that have already been addressed in the scientific community. It will also illustrate the direction that continued research will take us in the treatment and prevention of ROP.

\section{Pathogenesis of ROP}

It is necessary to understand the pathogenesis of ROP prior to exploring strategies for its prevention. ROP occurs when premature birth interrupts normal retinal vascular development [2]. In humans, normal retinal vasculature occurs in two phases: vasculogenesis and angiogenesis. Vasculogenesis is the de novo formation of blood vessels from endothelial precursor cells. Angiogenesis is the development of new blood vessels from existing blood vessels, which occurs from 17 - 18 weeks postmenstrual age, and is characterized by tissue hypoxia-driven blood vessel development mediated by Vascular Endothelial Growth Factor (VEGF) [2]. Given that physiologic hypoxia is the normal fetal state, ROP represents defective development of vasculature when the tissue environment is hyperoxic as compared to the in utero environment [2]. ROP itself is divided into two phases.

The first phase of ROP is a vaso-obliterative phase in which, due to the hyperoxic state of the premature infant, hypoxic stimulus for the secretion of VEGF by retinal astrocytes and Muller cells is stunted. This leads to a significant delay in endothelial cell angiogenesis and blood vessel development [2]. The second phase of ROP is a proliferative phase and begins at 32 - 34 weeks of postmenstrual age. It is characterized by a hypoxia-induced retinal neovascularization which can be explained by an imbalance between the poorly developed blood vessels and the increasing metabolic demands of developing neural retina [3]. Retinal hypoxia induces a molecular cascade that culminates in the expression of a variety of genes that encode for angiogenic growth factors, namely VEGF and IGF-1. These growth factors then participate in the pathological formation of blood vessels at the junction between the vascularized and nonvascularized retina. As is common in other proliferative retinopathies, pathological neovascularization can produce fibrous contraction which ultimately results in retinal traction, detachment, and possibly blindness [4].

\section{Oxygen}

We now know a great deal about the importance of physiologic hypoxia as a vital stimulus for VEGF in retinal vessel formation. It is thus not surprising that prolonged respiratory support and high pulse oximetry oxygen saturations have been associated with an increased risk of ROP [5]. It is important to note that the ideal target for oxygen saturation, in so far as it relates to the progression of ROP, varies depending on which phase of ROP is being studied. In phase I ROP, multiple studies have shown that keeping oxygen saturation lower than previously expected, resulted in a decreased incidence of ROP progression [5] [6]. The question that naturally follows is how low? In 2003 Chow et al implemented what was deemed an "oxygen management policy" with strict guidelines for use of FIO2 and monitoring oxygen saturation of neonates from the delivery room and throughout hospitalization. The researchers found the incidence of ROP progression from stage 3 to stage 4 to decrease from $12.5 \%$ to $2.5 \%$ in 4 years [7]. Wright et al. in 2006 published their results after implementation of a physiologic reduced oxygen protocol (PROP) in 3 NICUs across the country. They proposed that maintaining $\mathrm{SpO}_{2}$ values between $83 \%$ and $93 \%$ in the immediate postgestation life, combined with strict control of oxygen fluctuations, prevents the early vaso-obliterative phase of ROP [8]. A number of studies have since followed that have confirmed this trend in strict oxygen control, and have culminated in the Oxygen with Love (OWL) protocol first introduced by Sink et al in 2012 [9]. The protocol proposes several methods in maintaining the target O2 saturation of $85 \%$ - 93\% in very low birth weight infants from birth to 36 weeks post-menstrual age and then presents data showing a significant decline of ROP in their NICU. After education of neonatal physicians, nurses, and respiratory therapists on risks of hyperoxemia in neonatal infants and the use of standardized bedside oximeter alarm setting, the incidence of any ROP in this NICU dropped from $35 \%$ to $12 \%$. Furthermore, the incidence of ROP requiring ablative procedures dropped from $11 \%$ to $0 \%$ [9]. 
With regards to phase II ROP, the studies have been concerned with treating retinal hypoxia produced by the metabolic demand of growing the neural retina not met due to impaired vasculogenesis of phase I. Researchers proposed that treating retinal hypoxia in a premature infant in phase II with supplemental oxygen could prevent the continued increase in retinal VEGF and may help prevent the progression of proliferative neovascularization [10]. In 2000, the STOP-ROP trial was published looking at the rate of progression from prethreshold to threshold ROP in premature infants that were randomized to a conventional arm with pulse oximetry targeted at $89 \%$ to $94 \%$ saturation versus a supplemental arm with pulse oximetry targeted at $96 \%$ to $99 \%$ saturation. The rate of progression to threshold in at least 1 eye was $48 \%$ in the conventional arm and $41 \%$ in the supplemental arm [11]. Although low oxygen saturation appears to reduce the risk of severe ROP when carefully controlled within the first few weeks of life, the optimal level of saturation still remains uncertain [12]. This necessarily begs the question what other preventative measures can be implemented to prevent or at least modify the progression of ROP in premature births?

\section{Beta Blockers}

Beta $_{2}$-adrenergic receptors (beta 2 -ARs) are expressed on vascular endothelial cells, and there has been evidence that the stimulation of beta ${ }_{2}$-ARs can upregulate VEGF and subsequently promote neoangiogenesis in response to chronic ischemia [4]. In 2010, Filippi et al published a pilot study describing a protocol, PROP-ROP, to explore the possible therapeutic role of beta blockers in ROP. They demonstrated that in oxygen induced retinopathy (OIR) mice, proangiogenic factors were dose-dependently reduced by propranolol [4].

In an effort to apply animal model data to humans, a subsequent trial studied fifty-two preterm newborns with Stage 2 ROP who were randomized to receive oral propranolol in addition to standard treatment versus standard treatment alone [13]. They found that newborns treated with oral propranolol showed not only less progression to Stage 3 or Stage 3 plus but also that they required fewer laser treatments as well as less need for rescue treatment with intravitreal bevacizumab. These benefits, however, needed to be weighed against a number of serious adverse effects of systemic propranolol, such as hypotension and bradycardia, which were noted in 5 out of the 26 newborns treated with propranolol [13]. The use of oral propranolol was also studied by Makhoul et al in 2013 whose group determined that placebo infants required twice as many interventions as those treated with propranolol. However, due to the small number of participants, it was noted that the results were not statistically significant [14].

Given the concern for the potentially harmful systemic side effects of oral propranolol, the use of propranolol eye drops was first looked at in a pilot study by Dal Monti et al in 2013. They looked at topical applications of propranolol as related to prevention of retinal vascular proliferation in OIR mouse models [15]. In the first arm, the study demonstrated that topical propranolol did indeed reach the retina and resulted in drug concentrations that were effective. While these researchers admit that it might be difficult to extrapolate these results to humans, they were nonetheless excited to report that $\beta$-AR blockade could counteract oxygen induced retinopathy by preventing angiogenic responses to hypoxia. Topical propranolol could be a cheap, easy, and safe alternate delivery route to systemic propranolol [15]. Further trials are necessary to bring this data to the point of human application.

\section{Vitamin A}

In 2012, it was hypothesized that dark-adapted retinal sensitivity in preterm infants is improved by early high dose vitamin A [13]. This is explained by an increased availability of retinaldehyde for incorporation into rhodopsin in the developing retina. Mactier et al were the first to measure functional ocular stores of retinol in preterm infants, and found that early high-dose intramuscular vitamin A supplementation for infants at risk of retinopathy of prematurity improves retinal function at 36 weeks' post menstrual age [16]. However, the association between vitamin A and hypoxia-induced retinal neovascularization was not explored until Wang et al showed that systemic administration of retinoic acid in OIR mouse models can increase endogenous VEGF production. This, in turn, has beneficial effects on retinal vasculature and ultimately counteracts the first vaso-obliterative phase of ROP [17]. The reasons, as proposed by Wang and his colleagues, for strongly considering systemic vitamin A amongst the prevention strategies for ROP are compelling. Systemic application of retinoic acid is a more feasible mode of drug delivery in neonates than, for example, intravitreal administration. It also generates an endogenous supply of VEGF to the retina. Furthermore it should be noted that retinoic acid administration in extremely low birth weight 
infants has been shown to reduce the rate of bronchopulmonary dysplasia and associated pulmonary comorbidities. Finally, it is no small matter that the pharmacology, pharmacodynamics, and toxicology of retinoic acid in humans is well understood because it has been used safely and effectively in treating other conditions such as acute promyelocytic leukemia [17]. Clinical trials looking specifically at retinoic acid administration in neonates and the impact of progression of ROP promises to be an exciting area of research in the realm of ROP prevention.

\section{Vitamin E}

Since the 1940s, there have been a number of clinical trials of Vitamin E supplementation in premature infants to prevent ROP [10]. A Cochrane review of vitamin E studies concluded that supplemental vitamin E significantly reduced the risk of severe ROP in very low birth weight infants, however at the increased risk of sepsis [18]. One of these trials from the late 1980s found that the incidence of severe ROP was not significantly decreased; however they showed an increased incidence of sepsis and late-onset necrotizing enterocolitis (NEC) in Vitamin E-treated infants weighing less than or equal to $1500 \mathrm{gm}$ at birth who received study medication for greater than or equal to 8 days [19]. An additional study by Phelps et al. in 1987 documented increased incidence of hemorrhagic complications of prematurity, especially in infants weighing less than $1000 \mathrm{~g}$ [20]. It appears that above studies have come to a unanimous conclusion that the benefits of using vitamin $\mathrm{E}$ as prophylaxis against ROP do not come close to outweighing the risks of sepsis, NEC, and hemorrhagic complications.

\section{Serum IGF-1}

Scientists have postulated that in addition to oxygen related risk factors, non-oxygen related growth factors affected by prematurity itself are also known to play an important role in pathogenesis of ROP. It has been shown that lack of IGF-1, such as in IGF-1 knock out mice, is associated with the lack of vascular growth that subsequently leads to hypoxia-driven proliferative ROP [21]. Hellstrom et al subsequently applied this data to humans and showed that persistently low serum IGF-1 did indeed correlate with increased incidence of ROP. These authors went so far as to state that IGF-1 is as strong a predictor for ROP as is low birth weight and post menstrual age at birth [22]. In 2006, they established an algorithm called WINROP (Weight, Insulin-like growth factor I, Neonatal, Retinopathy of Prematurity) in which, IGF-I values can be used to screen for infants who might be at risk of ROP [23].

Scientists have explored methods at exogenous IGF-1 delivery to preterm infants. Human milk increases serum IGF-1 more than formula feeding, however given feeding intolerance that many premature infants face, there are efforts to increase IGF-1 by parenteral administration [10]. Fresh frozen plasma has been shown to be a source of exogenous IGF-1. Dani et al, in an effort to determine whether FFP infusions can decrease the rate of ROP, found that 2 or more transfusions of FFP in the first week of life decreased the risk of developing any grade of ROP in preterm infants [24].

\section{Omega-3 Supplements}

Supplementing omega-3-PUFA may be of benefit in preventing retinopathy. In 2007 Connor et al used a mouse model to study the influence of omega-3- and omega-6-polyunsaturated fatty acids (PUFAs) on vascular loss and hypoxia-induced neovascularization as it pertained to oxygen induced retinopathy. They successfully showed that increasing omega-3-PUFA served to increase vessel regrowth after injury, thereby decreasing the avascular area of the retina. This reduced the hypoxic stimulus for neovascularization [25].

It had been previously noted that DHA, a major long chain poly unsaturated fatty acid (LCPUF), is a fundamental structural component of retinal cells and that DHA concentration affects the enzyme activity of membranes of retinal photoreceptors and their function. DHA was noted to be necessary for optimal retinal function [26]. A metaanalyses recently published aimed to evaluate whether supplementation of infant formula LCPUFA before the age of 1 improves infants' visual acuity. Researches did find a significant benefit of LCPUFA supplementation to visual acuity, as assessed by visual evoked potentials and behavioral methods [26].

The impact of fortification of infant formula with DHA or supplementation with omega-3 fatty acids to breast-feeding mothers on phase I or phase II ROP in low birth weight infants needs further study [18]. If additional research does support this hypothesis, then an approach similar to folic acid prenatal supplementation can be undertaken [27]. 


\section{D-Pencillamine}

A known chelator in medicine, D-pencillamine, which had been used in prevention of hyperbilirubinemia in premature infants, was also thought to possibly contribute to lower incidence of ROP. In 2001, as part of a larger review, two randomized trials on the effects of prophylactic D-pencillamine on ROP were identified. When combined, they showed a significantly lower incidence of acute ROP in the treated infants [28].

Subsequent to these findings, as part of a Cochrane review, a study was conducted looking at outcome of ROP in controlled trials where D-penicillamine was administered as compared with no treatment or placebo. A meta-analysis of 3 randomized trials meeting inclusion criteria revealed that there was no significant difference in the risk of any stage of ROP. Based on this data the authors of the review could not recommend the use of D-penicillamine for the prevention of ROP [29].

\section{Inositol}

Human milk has many antioxidant constituents including inositol, vitamin E, and beta-carotene that may protect against the development of ROP. An observational cohort study was designed to look at the effect of human milk feedings on the incidence of ROP in infants weighing less than $1500 \mathrm{~g}$. It was determined that the incidence of ROP differed significantly by type of feeding i.e. human milk (41\%) vs. formula (63.5\%) [30].

This study paved the way for further research targeting specific nutrients such as inositol. In 2000, Friedman et al designed a prospective study looking at the relationship between inositol and ROP. They found that infants receiving high inositol formula and with higher serum inositol concentrations at birth and after 30 days had a statistically significant lower incidence of severe ROP than those receiving the lower inositol formula and with lower serum concentrations [31].

\section{Conclusion}

This brief review serves as a whirlwind look at preventative strategies for tackling ROP given what we already know about the pathogenesis of a potentially blinding disease. Certain strategies for prevention have been confirmed by numerous trials, namely the role of oxygen therapy as an independent risk factor for the development of ROP and the benefits of strict control of premature infant oxygen saturations in the NICU. There is valuable data that supports use of other approaches, such as beta adrenergic blockade and IGF-1 supplementation, for which clinical trials are still in the works to establish clear protocols for their clinical use. Supplementation with vitamin A, omega-3 fatty acids, and inositol are all exciting arenas for further trials as preliminary data shows promising results in ROP prevention. It has also been shown that the benefit of vitamin $\mathrm{E}$ is not worth the increased incidence of NEC and sepsis as potential side effects. Furthermore, while it was an interesting idea to use the chelator D-penicillamine as a preventative strategy for ROP, the results of multiple trials seemed to be equivocal. This brief review is not all-inclusive, as there are many more modes of prevention currently being studied. It is an exciting period in medicine as clinical trials continue to establish easier, safer, and more effective modes of prevention of ROP.

\section{References}

[1] National Eye Institute: Facts about Retinopathy of Prematurity (ROP). https://www.nei.nih.gov/health/rop/rop

[2] Fleck, B.W. and McIntosh, N. (2008) Pathogenesis of Retinopathy of Prematurity and Possible Preventive Strategies. Early Human Development, 84, 83-88. http://dx.doi.org/10.1016/j.earlhumdev.2007.11.008

[3] Penn, J.S., Madan, A., Caldwell, R.B., Bartoli, M., Caldwell, RW. and Hartnett, M.E. (2008) Vascular Endothelial Growth Factor in Eye Disease. Progress in Retinal and Eye Research, 27, 331-371. http://dx.doi.org/10.1016/j.preteyeres.2008.05.001

[4] Filippi, L., Cavallaro, G., Bagnoli, P., Dal Monte, M., Fiorini, P., Donzelli, G., Tinelli, F., Araimo, G., Cristofori, G., La Marca, G., Della Bona, M.L., La Torre, A., Fortunato, P., Furlanetto, S., Osnaghi, S. and Mosca, F. (2010) Study Protocol: Safety and Efficacy of Propranolol in Newborns with Retinopathy of Prematurity (PROP-ROP): ISRCTN18523491. BMC Pediatrics, 10, 83. http://dx.doi.org/10.1186/1471-2431-10-83

[5] Tin, W., Milligan, D.W., Pennefather, P. and Hey, E. (2001) Pulse Oximetry, Severe Retinopathy, and Outcome at One Year in Babies of Less than 28 Weeks Gestation. Archives of Disease in Childhood_Fetal and Neonatal Edition, 84, F106-F110. http://dx.doi.org/10.1136/fn.84.2.F106 
[6] VanderVeen, D.K., Mansfield, T.A. and Eichenwald, E.C. (2006) Lower Oxygen Saturation Alarm Limits Decrease the Severity of Retinopathy of Prematurity. Journal of AAPOS, 10, 445-448.

http://dx.doi.org/10.1016/j.jaapos.2006.04.010

[7] Chow, C., Wright, K.W. and Sola, A. (2003) Can Changes in Clinical Practice Decrease the Incidence of Severe Retinopathy of Prematurity in Very Low Birth Weight Infants? Pediatrics, 111, 339-345. http://dx.doi.org/10.1542/peds.111.2.339

[8] Wright, K.W., Sami, D., Thompson, L., Ramanathan, R. and Joseph, R. (2006) A Physiologic Reduced Oxygen Protocol Decreases the Incidence of Threshold Retinopathy of Prematurity. Transactions of the American Ophthalmological Society, 104, 78-84.

[9] Sink, D., Thomas, P., Bober, B. and Hagadom, J.I. (2012) Oxygen with Love (OWL) Improves Oxygen Saturation Target Achievement and Eye Outcomes in Preterm Newborn. http://www.docstoc.com/docs/73625317/OxygenwithLove_OWL_ImprovesOxygenSaturationTarget

[10] Raghuveer, T.S. and Bloom, B.T. (2011) A Paradigm Shift in the Prevention of Retinopathy of Prematurity. Neonatology, 100, 116-129. http://dx.doi.org/10.1159/000322848

[11] The STOP-ROP Multicenter Study Group (2000) Supplemental Therapeutic Oxygen for Prethreshold Retinopathy of Prematurity (STOP-ROP): A Randomized, Controlled Trial. I: Primary Outcome. Pediatrics, 105, 295-310.

[12] Cavallaro, G., Filippi, L., Bagnoli, P., La Marca, G., Cristofori, G., Raffaeli, G., Padrini, L., Araimo, G., Fumagalli, M., Groppo, M., Dal Monte, M., Osnaghi, S., Fiorini, P. and Mosca, F. (2014) The Pathophysiology of Retinopathy of Prematurity: An Update of Previous and Recent Knowledge. Acta Ophthalmologica, 92, 2-20.

[13] Filippi, L., Cavallaro, G., Bagnoli, P., Dal Monte, M., Fiorini, P., Donzelli, G., Tinelli, F., Araimo, G., Cristofori, G., la Marca, G., Della Bona, M.L., La Torre, A., Fortunato, P., Furlanetto, S., Osnaghi, S. and Mosca, F. (2013) Oral Propranolol for Retinopathy of Prematurity: Risks, Safety Concerns, and Perspectives. The Journal of Pediatrics, 163, 1570-1577. http://dx.doi.org/10.1016/j.jpeds.2013.07.049

[14] Makhoul, I.R., Peleg, O., Miller, B., Bar-Oz, B., Kochavi, O., Mechoulam, H., Mezer, E., Ulanovsky, I., Smolkin, T., Yahalom, C., Khoury, A., Lorber, A., Nir, A. and Blazer, S. (2013) Oral Propranolol versus Placebo for Retinopathy of Prematurity: A Pilot, Randomised, Double-Blind Prospective Study. Archives of Disease in Childhood, 98, 565-567. http://dx.doi.org/10.1136/archdischild-2013-303951

[15] Dal Monte, M., Casini, G., la Marca, G., Isacchi, B., Filippi, L. and Bagnoli, P. (2013) Eye Drop Propranolol Administration Promotes the Recovery of Oxygen-Induced Retinopathy in Mice. Experimental Eye Research, 111, 27-35. http://dx.doi.org/10.1016/j.exer.2013.03.013

[16] Mactier, H., McCulloch, D., Hamilton, R., Galloway, P., Bradnam, M.S., Young, D., Lavy, T., Farrell, L. and Weaver, L.T. (2012) Vitamin A Supplementation Improves Retinal Function in Infants at Risk of Retinopathy of Prematurity. The Journal of Pediatrics, 160, 954-959. http://dx.doi.org/10.1016/j.jpeds.2011.12.013

[17] Wang, L., Shi, P., Xu, Z., Li, J., Xie, Y., Mitton, K., Drenser, K. and Yan, Q. (2014) Up-Regulation of VEGF by Retinoic Acid during Hyperoxia Prevents Retinal Neovascularization and Retinopathy. Investigative Ophthalmology \& Visual Science, 55, 4276-4287. http://dx.doi.org/10.1167/iovs.14-14170

[18] Brion, L.P., Bell, E.F. and Raghuveer, T.S. (2003) Vitamin E Supplementation for Prevention of Morbidity and Mortality in Preterm Infants. Cochrane Database of Systematic Reviews, Article ID: CD003665. http://dx.doi.org/10.1002/14651858.cd003665

[19] Johnson, L., Quinn, G.E., Abbasi, S., Otis, C., Goldstein, D., Sacks, L., Porat, R., Fong, E., Delivoria-Papadopoulos, M., Peckham, G., et al. (1989) Effect of Sustained Pharmacologic Vitamin E Levels on Incidence and Severity of Retinopathy of Prematurity: A Controlled Clinical Trial. The Journal of Pediatrics, 114, 827-838. http://dx.doi.org/10.1016/S0022-3476(89)80149-0

[20] Phelps, D.L., Rosenbaum, A.L., Isenber, S.J., Leake, R.D. and Dorey, F.J. (1987) Tocopherol Efficacy and Safety for Preventing Retinopathy of Prematurity: A Randomized, Controlled, Double-Masked Trial. Pediatrics, 79, 489-500.

[21] Smith, L.E.H. (2008) Through the Eyes of a Child: Understanding Retinopathy through ROP the Friedenwald Lecture. Investigative Ophthalmology \& Visual Science, 49, 5177-5182. http://dx.doi.org/10.1167/iovs.08-2584

[22] Hellström, A., Engström, E., Hård, A.L., Albertsson-Wikland, K., Carlsson, B., Niklasson, A., Löfqvist, C., Svensson, E., Holm, S., Ewald, U., Holmström, G. and Smith, L.E. (2003) Postnatal Serum Insulin-Like Growth Factor I Deficiency Is Associated with Retinopathy of Prematurity and Other Complications of Premature Birth. Pediatrics, 112, 1016-1020. http://dx.doi.org/10.1542/peds.112.5.1016

[23] Löfqvist, C., Engström, E., Sigurdsson, J., Hard, A.L., Niklasson, A., Ewald, U., et al. (2006) Postnatal Head Growth Deficit among Premature Infants Parallels Retinopathy of Prematurity and Insulin-Like Growth Factor-I Deficit. Pediatrics, 117, 1930-1938. http://dx.doi.org/10.1542/peds.2005-1926

[24] Dani, C., Poggi, C., Bresci, C., Corsini, I., Frosini, S. and Pratesi, S. (2014) Early Fresh-Frozen Plasma Transfusion 
Decreases the Risk of Retinopathy of Prematurity. Transfusion, 54, 1002-1007. http://dx.doi.org/10.1111/trf.12432

[25] Connor, K.M., SanGiovanni, J.P., Lofqvist, C., Aderman, C.M., Chen, J., Higuchi, A., et al. (2007) Increased Dietary Intake of $\omega$-3-Polyunsaturated Fatty Acids Reduces Pathological Retinal Angiogenesis. Nature Medicine, 7, 868-873. http://dx.doi.org/10.1038/nm1591

[26] Qawasmi, A., Landeros-Weisenberger, A. and Bloch, M.H. (2013) Meta-Analysis of LCPUFA Supplementation of Infant Formula and Visual Acuity. Pediatrics, 131, e262-e272. http://dx.doi.org/10.1542/peds.2012-0517

[27] Mantagos, I.S., Vanderveen, D.K. and Smith, L.E. (2009) Emerging Treatments for Retinopathy of Prematurity. Seminars in Ophthalmology, 24, 82-86. http://dx.doi.org/10.1080/08820530902800322

[28] Phelps, D.L., Lakatos, L. and Watts, J.L. (2001) D-Penicillamine for Preventing Retinopathy of Prematurity in Preterm Infants. Cochrane Database of Systematic Reviews, Article ID: CD001073. http://dx.doi.org/10.1002/14651858.cd001073

[29] Qureshi, M.J. and Kumar, M. (2013) D-Penicillamine for Preventing Retinopathy of Prematurity in Preterm Infants. Cochrane Database of Systematic Reviews, 9, Article ID: CD001073. http://dx.doi.org/10.1002/14651858.cd001073.pub2

[30] Hylander, M.A., Strobino, D.M., Pezzullo, J.C. and Dhanireddy, R. (2001) Association of Human Milk Feedings with a Reduction in Retinopathy of Prematurity among Very Low Birth-Weight Infants. Journal of Perinatology, 21, 356362. http://dx.doi.org/10.1038/sj.jp.7210548

[31] Friedman, C.A., McVey, J., Borne, M.J., James, M., May, W.L., Temple, D.M., Robbins, K.K., Miller, C.J. and Rawson, J.E. (2000) Relationship between Serum Inositol Concentration and Development of Retinopathy of Prematurity: A Prospective Study. Journal of Pediatric Ophthalmology \& Strabismus, 37, 79-86. 